\title{
Identification of Nonstandard Multifractional Brownian Motions under White Noise by Multiscale Local Variations of Its Sample Paths
}

\author{
Kwang-Il Ahn and Kichun Lee \\ Industrial Engineering Department, Hanyang University, 222 Wangsimni-ro, Seongdong-gu, Seoul 133-791, Republic of Korea \\ Correspondence should be addressed to Kichun Lee; skylee@hanyang.ac.kr
}

Received 18 July 2013; Accepted 9 August 2013

Academic Editor: Ming Li

Copyright (C) 2013 K.-I. Ahn and K. Lee. This is an open access article distributed under the Creative Commons Attribution License, which permits unrestricted use, distribution, and reproduction in any medium, provided the original work is properly cited.

\begin{abstract}
The Hurst exponent and variance are two quantities that often characterize real-life, high-frequency observations. Such real-life signals are generally measured under noise environments. We develop a multiscale statistical method for simultaneous estimation of a time-changing Hurst exponent $H(t)$ and a variance parameter $C$ in a multifractional Brownian motion model in the presence of white noise. The method is based on the asymptotic behavior of the local variation of its sample paths which applies to coarse scales of the sample paths. This work provides stable and simultaneous estimators of both parameters when independent white noise is present. We also discuss the accuracy of the simultaneous estimators compared with a few selected methods and the stability of computations with regard to adapted wavelet filters.
\end{abstract}

\section{Introduction}

Fractional Brownian motion ( $\mathrm{fBm}$ ) has been commonly used to characterize a wide range of complex signals in natural phenomena that exhibit self-similarity and longrange dependence since the pioneering work of Mandelbrot and Van Ness [1]. Examples of such complex signals in time are abundant in medicine, economics, and geoscience, to list a few. The $\mathrm{fBm}$ model is characterized by two parameters of the regularity level and the variance level of a signal. The regularity attribute, also called the Hurst exponent, expresses the strength of statistical similarity at many different frequencies, and the variance attribute describes an order of energy magnitude.

To model path regularity varying with time, multifractional Brownian motion $(\mathrm{mBm})$ has been proposed as a generalization of fractional Brownian motion $(\mathrm{fBm})$. The theory and applications of both $\mathrm{fBm}$ and $\mathrm{mBm}$ models have attracted the interests of researchers in numerous problems of, for example, sea level fluctuations [2], currency exchange rates [3], and network traffic [4-6]. To model mBm, LévyVéhel and Peltier [7] proposed a mean average approach, and Benassi et al. [8] introduced a spectral approach. Lim and Muniandy $[9,10]$ also proposed a mBm model based on the $\mathrm{fBm}$ defined by the Riemann-Liouville type of fractional integral. The proposed models represent $\mathrm{mBm}$ as a Gaussian process $W(t)$ with a covariance function involving Hurst exponent $H$ by a function of time, $H(t)$, and variance parameter $C$. Specifically, a Gaussian process $(W(t))_{t \geq 0}$ is called $\mathrm{mBm}$ with Hurst function $H(t)$ and its variance level (scaling factor) $C$ if its covariance function is represented as

$$
\begin{aligned}
\mathbb{E}[W(t) W(s)] \\
=\frac{C^{2}}{2} g(H(t), H(s)) \\
\quad \times\left\{|t|^{H(t)+H(s)}+|s|^{H(t)+H(s)}-|t-s|^{H(t)+H(s)}\right\}
\end{aligned}
$$

for $H \in \mathbb{C}^{\eta}([0,1]) ; t, s \in[0,1] ; g(x, y)=\sqrt{K(2 x) K(2 y)} /$ $K(x+y)$; and $K(\alpha)=\Gamma(\alpha+1) \sin (\alpha \pi / 2) / \pi, 0<\alpha<2$. The process is well defined, or square-integrable, if function $H(t)$ is the Hölderian of order $0<\eta \leq 1$ on $[0,1]$. Clearly, the process $W(t)$ is not weakly stationary since the covariance function $\mathbb{E}[W(t) W(s)]$ does not depend on $t-s$ only. From (1), we have $\mathbb{E}\left[W(t)^{2}\right]=C^{2} t^{H(t)}$, and consequently, $\operatorname{Var}[W(1)]=$ $C^{2}$. In this sense, $C$ is called the variance level of the process. 
The time-changing Hurst exponent $H(t)$ characterizes the path regularity of process $W$ at time $t$ since sample paths near $t$ with small $H(t)$, close to 0 , are space filling and highly irregular, while paths with large $H(t)$, close to 1 , are very smooth. The variance constant $C$ determines the energy level of the process. Alternatively, a spectral representation of $\mathrm{mBm}$ is given by

$$
W(t)=C \sqrt{\frac{K(2 H(t))}{2}} \int_{\mathbb{R}} \frac{e^{\mathrm{i} t \lambda}-1}{|\lambda|^{H(t)+1 / 2}} d B(\lambda),
$$

where $C$ is a constant scale (variance) parameter and $B$ the standard Brownian [7].

Several approaches were proposed to estimate timechanging Hurst exponent $H(t)$ and variance $C$ from sample paths of $\mathrm{mBm}$ signals. Benassi et al. [8] investigated estimation of a continuously differentiable $H(t)$ without the direct estimation of $C$. A local version of quadratic variations was used in several researches to estimate the constant Hurst exponent [11-13]. Recently Fhima et al. [14] adopted the increment ratio statistic method for $H(t)$ estimation only. For an overview of estimating constant $H(t)$, the reader is also referred to Beran [15] including various statistical methods or Bardet and Bertrand [16] concentrating on wavelet approaches. Estimation of both $H(t)$ and variance parameter $C$ has received little attention from the statistics community while $C$ is mostly treated as a nuisance parameter. When a signal is modeled with $\mathrm{mBm}$, the estimation of $H(t)$ can be improved by the accurate estimation of $C$ from covariance structures involving both $H(t)$ and $C$. For that purpose, the application of a local version of quadratic variations for estimating $H(t)$ and $C$ in $\mathrm{mBm}$ was discussed in Coeurjolly [17], in which $C$ was, however, locally estimated in each sample path. Moreover, the existence of noise in $\mathrm{mBm}$ signals has not been dealt with in the literature though real-life signals are commonly measured under noise environments.

The main objective of this paper is to develop a stable and accurate estimation procedure for unknown parameters $(H(t), C)$ given a path of $W(t)$ in the presence of independent white noise. Previous approaches by Coeurjolly [17] relied on local sample paths in the absence of white noise that resulted in estimators of $C$ sensitive to the sampled paths. It is widely accepted that noise occurs from a variety of sources such as measurement devices.

In this paper, we assume that $\mathrm{mBm}$ signals are contaminated by a moderate amount of noise. We extend the quadratic variations method to estimate $H(t)$ and $C$ simultaneously for $\mathrm{mBm}$ by applying dilated high-pass filters to all sampled paths (all subsample paths from a given sample path) and aggregating all local conditions from the previous filtering step. This method includes filtering all sampled paths with a dilated filter possessing a sufficient number of vanishing moments to capture regularity conditions at associated coarse scales and generating stationary filtered signals. The method further calculates empirical moments of the filtered signals and then estimates $H(t)$ and $C$ simultaneously together with a noise level in a regression setup specified by the empirical moments.

This paper is organized as follows. Section 2 introduces local variations in a $\mathrm{mBm}$ setting, discussing the procedures and justification for the simultaneous estimators of unknown parameters. Section 3 discusses numerical simulations and computational issues with adapted wavelet filters. The appendix presents proofs for the propositions in the preceding sections.

\section{Multiscale Local Variations of Multifractional Brownian Motion}

Let us consider a case in which a discretized sample path $\left(\mathbf{W}^{\prime}\right)$ is given by

$$
W^{\prime}\left(\frac{i}{N}\right)=W\left(\frac{i}{N}\right)+\sigma \varepsilon\left(\frac{i}{N}\right), \quad i=1, \ldots, N,
$$

in which $\varepsilon(i / N)$ is independent white noise and $\sigma$ is the noise level. Hurst function $H(t)$, generated by $W(i / N)$, is assumed to be Hölderian function of order $0<\eta \leq 1$ on $[0,1]$. In addition, noise magnitude $\sigma$ is assumed to be sufficiently small compared to the variance of $\mathrm{mBm}$. The covariance function of $\left(\mathbf{W}^{\prime}\right)$ is

$$
\begin{aligned}
\mathbb{E}\left[W^{\prime}(t) W^{\prime}(s)\right]= & \frac{C^{2}}{2} g(H(t), H(s)) \\
& \times\left\{|t|^{H(t)+H(s)}+|s|^{H(t)+H(s)}\right. \\
& \left.\quad-|t-s|^{H(t)+H(s)}\right\}+\sigma^{2} 1 \quad(t=s),
\end{aligned}
$$

where $\mathbf{1}(A)$ is an indicator of relation $A$ and $g(x, y)=$ $\sqrt{K(2 x) K(2 y)} / K(x+y)$ as defined above. From the above covariance function, we have $\mathbb{E}\left[W(t)^{2}\right]=C^{2} t^{2 H(t)}+\sigma^{2}$, and consequently, $\operatorname{Var}[W(1)]=C^{2}+\sigma^{2}$. Noticeably the estimation of $C$ is nontrivial because of the dependence structure from the covariance function; that is to say, the sample variance of a sample path does not lead to the direct expression of $C$ alone but to an expression mixed with all unknown parameters. The entries in (4) generate covariance matrix $\Sigma$, which depends on unknown parameters $\theta:=$ $(H(t), C, \sigma) \in R^{N+2}$. The covariance matrix consists of $N(N+$ 1)/2 parameters (due to symmetry) that can be organized into an $N(N+1) / 2 \times 1$ vector $\Gamma(\theta)$. Model (4) is locally identifiable almost everywhere if Jacobian matrix $\partial \Gamma(\theta) / \partial \theta^{\prime}$, which is $N(N+1) / 2 \times(N+2)$, has full column rank [18].

In order to weaken the dependence in $W^{\prime}(t)$ in (3), a differencing filter a of length $l+1$ and order $p>1$ (the number of vanishing moments) is applied. Filter $\mathbf{a}$ is defined by its taps $\left(a_{0}, \ldots, a_{l}\right)$ such that

$$
\begin{gathered}
\sum_{q=0}^{l} a_{q} q^{i}=0, \quad i=0, \ldots, p-1, \\
\sum_{q=0}^{l} a_{q} q^{i} \neq 0, \quad i=p .
\end{gathered}
$$


Let us also introduce $\mathbf{a}^{(m)}$ based on filter $\mathbf{a}$, defined by

$$
a_{i}^{(m)}= \begin{cases}a_{i / m}, & \frac{i}{m} \text { is an integer } \\ 0, & \text { otherwise. }\end{cases}
$$

We observe that $\mathbf{a}^{(m)}$, the filter a dilated $m$ times, focuses on a resolution at a low frequency, corresponding to a coarse space, as $m$ increases. For $m=1$, it captures the finest level of detail. For example, $\mathbf{a}^{(1)}=\mathbf{a}$ by definition, and for a second-order filter $\mathbf{a}:=(1,-2,1)$, $\mathbf{a}^{(2)}$ becomes $(1,0,-2,0,1)$. Furthermore, we can choose a as high-pass wavelet filters corresponding to orthogonal wavelets such as Daubechies and Symlet wavelets. A detailed discussion of wavelet filters can be found in Daubechies [19] and Vidakovic [20].

Let $\left(\mathbf{V}_{\mathbf{a}^{(m)}}^{\prime}\right)$ be a process consisting of $\left(\mathbf{W}^{\prime}\right)$ filtered by $\mathbf{a}^{(m)}$, that is,

$$
V_{\mathbf{a}^{(m)}}^{\prime}\left(\frac{j}{N}\right)=\sum_{q=0}^{m l} a_{q}^{(m)} W^{\prime}\left(\frac{j-q}{N}\right), \quad \text { for } j=m l+1, \ldots, N .
$$

For example, when a is $(1,-2,1)$, the filter is of order 2 , and $\left(V_{\mathbf{a}}^{\prime}\right)$ represents the second-order differences of $\left(W^{\prime}\right)$. The process $\left(\mathbf{V}_{\mathbf{a}^{(m)}}\right)$ is defined similarly with $(\mathbf{W})$ instead of $\left(\mathbf{W}^{\prime}\right)$ : $\left(\mathbf{V}_{\mathbf{a}^{(m)}}\right)$ is a process consisting of $(\mathbf{W})$ filtered by $\mathbf{a}^{(m)}$. The filtering by $\mathbf{a}^{(m)}$ breaks the dependence structure between observations. Specifically the process $\left(\mathbf{V}_{\mathbf{a}^{(m)}}^{\prime}\right)$ is stationary due to the vanishing moment property of filter $\mathbf{a}^{(m)}$. To verify it, we need to introduce a sufficiently small neighborhood covering $j$. Let $\nu(t)$ be an index set of a neighborhood of $t$, defined as

$$
\nu(t)=\left\{j \in \mathbb{Z}|l<j \leq N,| \frac{j}{N}-t \mid \leq \epsilon\right\}
$$

for a parameter $\epsilon>0$. We set $\epsilon$ to be a function of $N$ in such a way that $\epsilon \rightarrow 0, \epsilon N \rightarrow \infty$, and $\epsilon^{\eta} \log (N) \rightarrow 0$ as $N \rightarrow \infty$. In other words, for a sufficiently large $N$, the size of one neighbor becomes sufficiently small while maintaining the summation of the sizes of all the neighbors that are sufficiently large. In addition, it is possible to make $\epsilon^{\eta}$ converge to zero faster than $\log (N)$ grows. Then we derive the covariance of $\left(\mathbf{V}_{\mathbf{a}^{(m)}}^{\prime}\right)$ as follows.

Proposition 1. Let $j_{1} \in v\left(t_{1}\right), j_{2} \in v\left(t_{2}\right)$. Then, the covariance of $V_{\mathbf{a}^{(m)}}^{\prime}(j / N)$ in $(7), \mathbb{E}\left[V_{\mathbf{a}^{(m)}}^{\prime}\left(j_{1} / N\right) V_{\mathbf{a}^{(m)}}^{\prime}\left(j_{2} / N\right)\right]$, depends on $j_{1}-j_{2}$ as follows:

$$
\begin{aligned}
& \mathbb{E}\left[V_{\mathbf{a}^{(m)}}^{\prime}\left(\frac{j_{1}}{N}\right) V_{\mathbf{a}^{(m)}}^{\prime}\left(\frac{j_{2}}{N}\right)\right] \\
& =\frac{C^{2} g\left(H\left(t_{1}\right), H\left(t_{2}\right)\right)}{N^{H\left(t_{1}\right)+H\left(t_{2}\right)}} \\
& \quad \times \pi_{\mathbf{a}^{(m)}, H\left(t_{1}\right) / 2+H\left(t_{2}\right) / 2}\left(j_{1}-j_{2}\right) \\
& +\sigma^{2} \sum_{p-q=j_{1}-j_{2}} a_{p}^{(m)} a_{q}^{(m)}+\mathcal{O}\left(\epsilon^{\eta} \log N\right),
\end{aligned}
$$

where

$$
\pi_{\mathbf{a}^{(m)}, h}(k)=-\frac{1}{2} \sum_{p, q=0}^{m l} a_{p}^{(m)} a_{q}^{(m)}|k-p+q|^{2 h}
$$

The above proposition states that $V_{\mathbf{a}^{(m)}}^{\prime}(j / N)$ is weakly stationary as Gaussian. Particularly for $j_{1}=j_{2}=j$, as $N \rightarrow \infty$, it simplifies to

$$
E\left[V_{\mathbf{a}^{(m)}}^{\prime}\left(\frac{j}{N}\right)^{2}\right]=C^{2}\left(\frac{m}{N}\right)^{2 H(t)} \pi_{\mathbf{a}, H(t)}(0)+\sigma^{2} \sum_{q} a_{q}^{2} .
$$

Observably the above proposition deals with two pointwise positions, $j_{1}$ and $j_{2}$, for two neighborhoods near $t_{1}$ and $t_{2}$, respectively. Thus an aggregate behavior of each neighborhood is analyzed via the following setup.

Let us define the second empirical moment of the filtered signal $V_{\mathbf{a}^{(m)}}^{\prime}$ as follows:

$$
S^{\prime}\left(t, \mathbf{a}^{(m)}\right)=\frac{1}{|\nu(t)|} \sum_{j \in \nu(t)} V_{\mathbf{a}^{(m)}}^{\prime}\left(\frac{j}{N}\right)^{2}, \quad \text { for } t \in[0,1],
$$

which represents the average squared energy of the $\mathbf{a}^{(m)}$ filtered signal in the neighborhood of $t$. We notice that $S^{\prime}\left(t, \mathbf{a}^{(m)}\right)$ is random because $V_{\mathbf{a}^{(m)}}^{\prime}(j / N)$ is random and its expectation $E\left[S^{\prime}\left(t, \mathbf{a}^{(m)}\right)\right]$ equals that of $V_{\mathbf{a}^{(m)}}^{\prime}(j / N)^{2}$ because $V_{\mathbf{a}^{(m)}}^{\prime}(j / N)$ is weakly stationary. That is to say,

$$
\begin{aligned}
E\left[S^{\prime}\left(t, \mathbf{a}^{(m)}\right)\right] & =E\left[V_{\mathbf{a}^{(m)}}^{\prime}\left(\frac{j}{N}\right)^{2}\right] \\
& =C^{2}\left(\frac{m}{N}\right)^{2 H(t)} \pi_{\mathbf{a}, H(t)}(0)+\sigma^{2} \sum_{q} a_{q}^{2} .
\end{aligned}
$$

Now, to relate $S^{\prime}\left(t, \mathbf{a}^{(m)}\right)$ to $E\left[S^{\prime}\left(t, \mathbf{a}^{(m)}\right)\right]$ more specifically, we define a statistic $V\left(t, \mathbf{a}^{(m)}\right)$, called the $m$-scale local variation of $(\mathbf{W})$, as

$$
V\left(t, \mathbf{a}^{(m)}\right)=\frac{1}{|\nu(t)|} \sum_{j \in \nu(t)}\left\{\frac{V_{\mathbf{a}^{(m)}}(j / N)^{2}}{\mathbb{E}\left[V_{\mathbf{a}^{(m)}}(j / N)^{2}\right]}-1\right\},
$$

where $|\nu(t)|$ is the cardinal number of $\nu(t)$. The statistic $V\left(t, \mathbf{a}^{(m)}\right)$ captures the amount of deviations of the $\mathbf{a}^{(m)}$ filtered signal from the standard normal distribution near $t$ because $V_{\mathbf{a}^{(m)}}$ is normalized by its standard deviation, the square root of $\mathbb{E}\left[V_{\mathbf{a}^{(m)}}(j / N)^{2}\right]$. The definition based on the second order can be extended to the $k$ th order Hermite polynomial in the summation of (14): the second Hermite polynomial is defined by $t^{2}-1$. In this paper, we use local variations based on the second Hermite polynomial as the minimum asymptotic variance estimators, as shown in Coeurjolly [13] for fBm settings. Next, we connect the $m$ scale local variation $V\left(t, \mathbf{a}^{(m)}\right)$ with the empirical moment $S^{\prime}\left(t, \mathbf{a}^{(m)}\right)$ through the following relationship. 


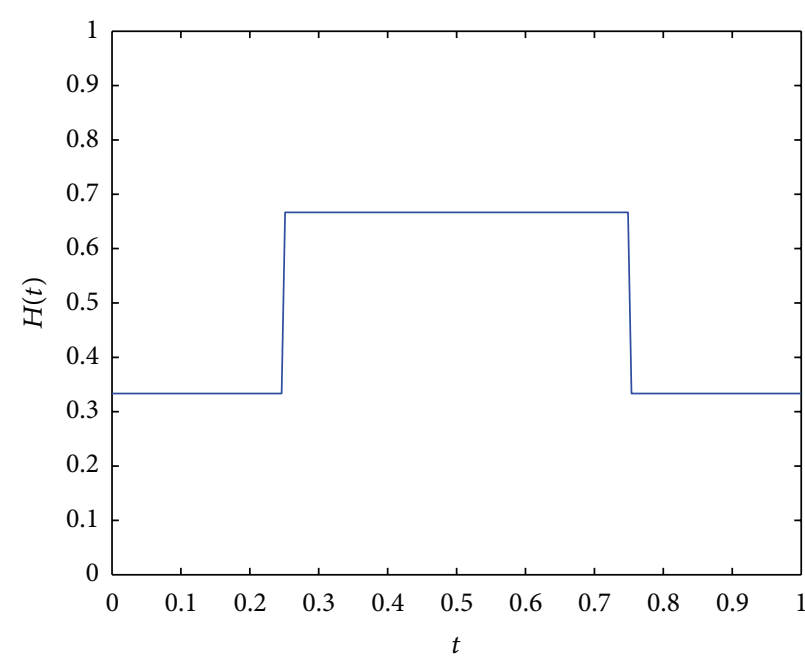

(a)

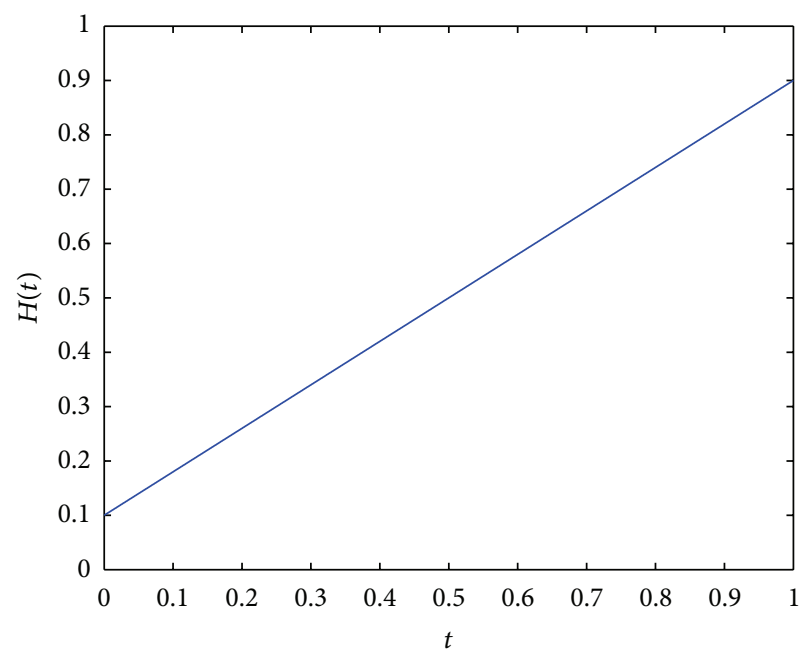

(c)

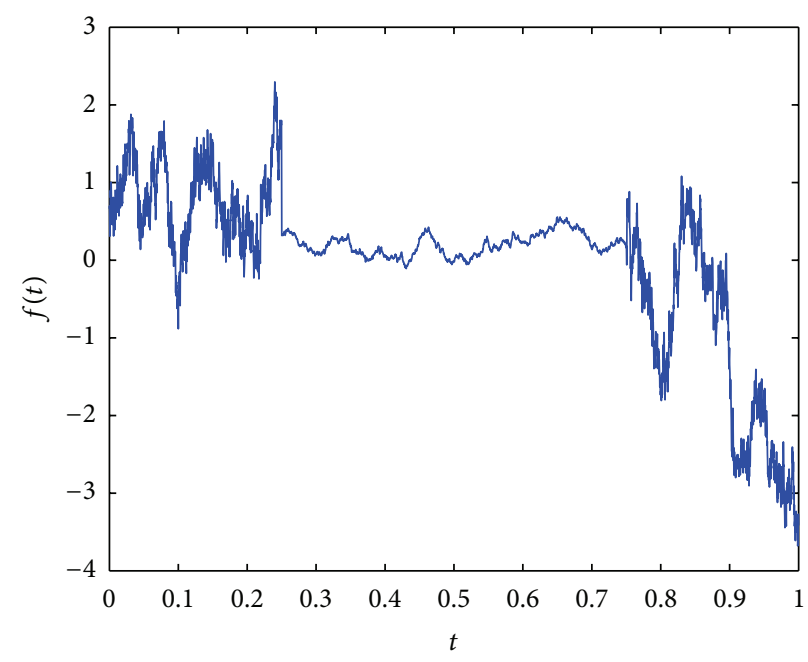

(b)

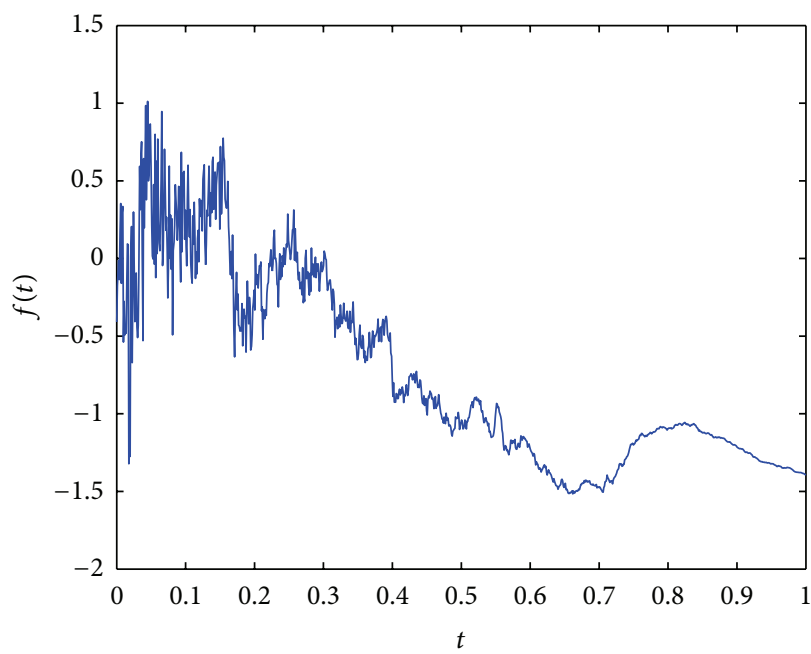

(d)

FIGURE 1: Tested Hurst functions are shown in (a) step-function $H(t)$ and (c) straight-line $H(t)$; their illustrations of signals are shown in (b) and (d), correspondingly.

Proposition 2. Let $m$-scale local variation $V\left(t, \mathbf{a}^{(m)}\right)$ and the empirical moment $S^{\prime}\left(t, \mathbf{a}^{(m)}\right)$ be defined by (14) and (12), respectively, given $W^{\prime}(t)$ as above and a of order $>1$. Then

$$
\begin{array}{r}
\log S^{\prime}\left(t, \mathbf{a}^{(m)}\right)=\log \mathbb{E}\left[S^{\prime}\left(t, \mathbf{a}^{(m)}\right)\right]+V\left(t, \mathbf{a}^{(m)}\right)(1+o(1)), \\
\text { as } N \longrightarrow \infty .
\end{array}
$$

The proposition connects the empirical moment $S^{\prime}\left(t, \mathbf{a}^{(m)}\right)$ and the log of its expectation through $m$-scale local variation $V\left(t, \mathbf{a}^{(m)}\right)$. Since the $m$-scale local variation converges almost surely to 0 and its distribution follows normal distribution asymptotically [17], the above relationship establishes a regression setup. We also note that a filter of an order of at least 2 ensures asymptotic normality for all the values of the function $H(t)$. For a filter of order 1 , this convergence is available if and only if $0<\sup _{t} H(t)<3 / 4$.
Next the relationship between the log of the expectation of the empirical moment $S^{\prime}\left(t, \mathbf{a}^{(m)}\right)$ and the parameters of interest $(H(t), C$, and $\sigma)$ is derived naturally in the light of Proposition 2 and (13). Thus for $t_{1}, \ldots, t_{d} \in[0,1]$ we obtain a regression model for $\log S^{\prime}\left(t_{i}, \mathbf{a}^{(m)}\right)$ as $N \rightarrow \infty$ :

$$
\begin{array}{r}
\log S^{\prime}\left(t_{i}, \mathbf{a}^{(m)}\right) \sim \log \left(C^{2}\left(\frac{m}{N}\right)^{2 H\left(t_{i}\right)} \pi_{\mathbf{a}, H\left(t_{i}\right)}(0)+\sigma^{2} \sum_{q} a_{q}^{2}\right), \\
\forall i, m,
\end{array}
$$

which is nonlinear with respect to $H\left(t_{i}\right), C$, and $\sigma$. In particular, when the noise level $\sigma$ is considered to be zero, the regression model simplifies to, for all $i$ and $m$,

$$
\begin{aligned}
\log S^{\prime}\left(t_{i}, \mathbf{a}^{(m)}\right) \sim & 2 \log C+2 H\left(t_{i}\right) \log \left(\frac{m}{N}\right) \\
& +\log \left(\pi_{\mathbf{a}, H\left(t_{i}\right)}(0)\right)
\end{aligned}
$$




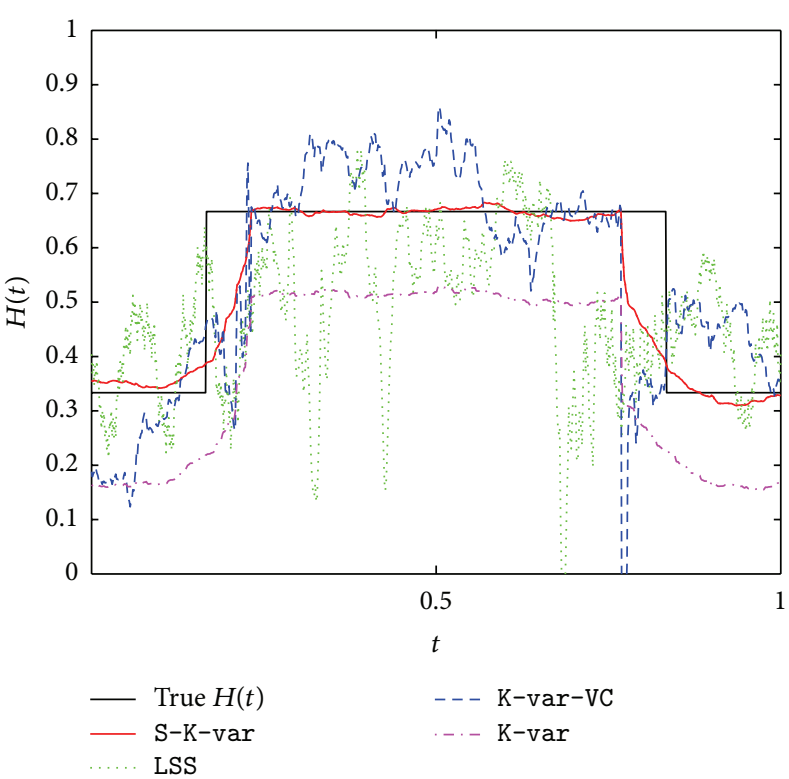

(a)

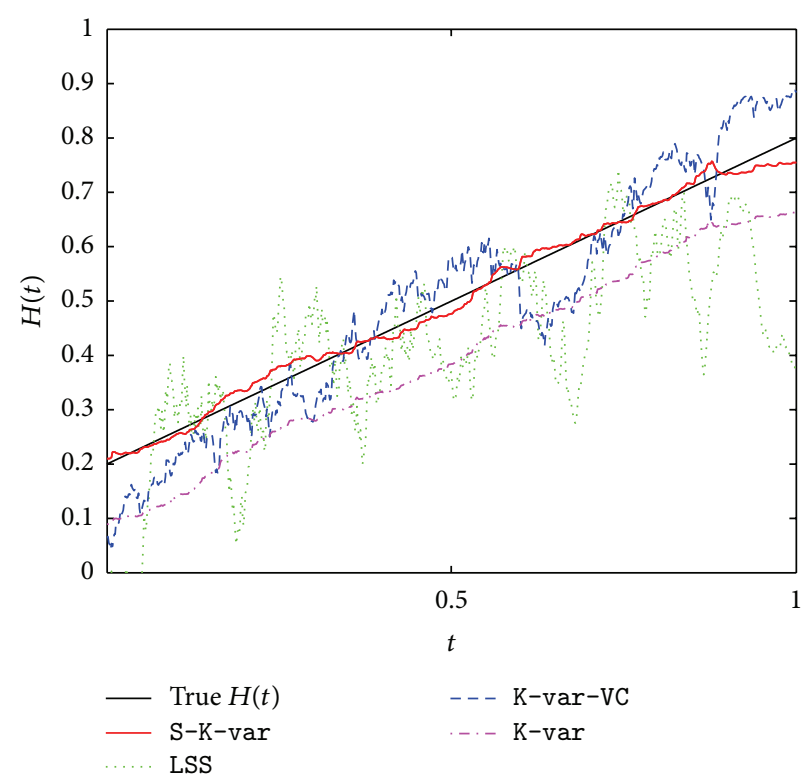

(b)

Figure 2: Illustrations of the estimators: in panel (a), variance $C$ was 2; in panel (b), variance $C$ was 4 ; the best match to the true $H(t)$ was the method of $\mathrm{S}-\mathrm{K}$-var among the four.

$$
\begin{aligned}
= & {\left[\begin{array}{ll}
1 & 2 \log \left(\frac{m}{N}\right)
\end{array}\right] } \\
& \times\left[\begin{array}{c}
2 \log C+\log \left(\pi_{\mathbf{a}, H\left(t_{i}\right)}(0)\right) \\
H\left(t_{i}\right)
\end{array}\right],
\end{aligned}
$$

which turns out to be linear with respect to $H\left(t_{i}\right)$ with intercept $2 \log C$, if $\log \left(\pi_{\mathbf{a}, H\left(t_{i}\right)}(0)\right)$ is negligible. The above regression model possesses a computational advantage though ignoring the presence of noise.

When $\sigma$ is nonzero, the following least square estimator of $(\mathbf{H}, C, \sigma)$ is introduced:

$$
\begin{aligned}
(\widehat{\mathbf{H}}, \widehat{C}, \widehat{\sigma})=\underset{(\mathbf{H}, C, \sigma)}{\arg \min } & \\
& \times \sum_{i=1}^{d} \sum_{m=1}^{M}\left(\log S\left(t_{i}, \mathbf{a}^{(m)}\right)-\log \mathbb{E}\left[S\left(t_{i}, \mathbf{a}^{(m)}\right)\right]\right)^{2} .
\end{aligned}
$$

The computation of the least-square estimator is feasible because, based on (16), for fixed $C$ as $C_{o}$ and $\sigma$ as $\sigma_{o}$, the computation of $\mathbf{H}$ is separable into each $H\left(t_{i}\right)$. In other words, a solution of $H\left(t_{i}\right)$ is given by

$$
\begin{aligned}
\widehat{H}\left(t_{i}\right)=\underset{h}{\arg \min } & \\
& \times \sum_{m=1}^{M}\left(\log S^{\prime}\left(t_{i}, \mathbf{a}^{(m)}\right)\right. \\
& \left.\quad-\log \left(C_{o}^{2}\left(\frac{m}{N}\right)^{2 h} \pi_{\mathbf{a}, h}(0)+\sigma_{o}^{2} \sum_{q} a_{q}^{2}\right)\right)^{2} .
\end{aligned}
$$

Numerical approaches such as the bisection method can be used for the above procedure, which is nonlinear in $h$. The bisection method achieves a desired precision level, $\tau$, for $\widehat{H}\left(t_{i}\right)$ with the number of iterations greater than $\log _{2} \tau^{-1}$. In other words, 10 iterations, for example, results in precision $\tau<0.001$.

\section{Simulations and Comparisons}

We present here a simulation study of the performance of the approach suggested in this paper, denoted by $\mathrm{S}-\mathrm{K}-\mathrm{var}$. Simulation is done with the "known truth" of Hurst function $H(t)$ the controlled signal variance and the signal-to-noise (SNR) ratio. Test functions are shown in Figure 1 with the step function for $H(t)$ in Figure $1(\mathrm{a})$ and the straight-line function in Figure 1(c). Their illustrations of $W^{\prime}(t)$ are shown in Figures 1(b) and 1(d), correspondingly. For the sake of comparison, we chose several popular methods such as the local spectra slope, which is summarized in Gao [21] and denoted by LSS, and $k$-variation of variance-uncorrected, denoted by $\mathrm{K}$-var, and the $k$-variation of variance-corrected version in Coeurjolly [13], denoted by K-var-VC. The average mean squared error (AMSE) was used as a performance measure to capture the difference between true $H(t)$ and estimated $\widehat{H}(t)$. To simulate a sample path from a $\mathrm{fBm}$ on $[0,1]$, we used the method of Wood and Chan [22]. One can simulate a standard $\mathrm{mBm} W$ with covariance matrix $C_{H(\cdot)}$ by generating $Z \sim N\left(0, I_{N}\right)$ and estimating $W:=C_{H(\cdot)}^{1 / 2} Z$. This method is exact in theory and sufficiently fast for a reasonable sample size $N$.

In this section we will use the following notations regarding filters: Diff . i denoting the filter of differences of order $i$, 


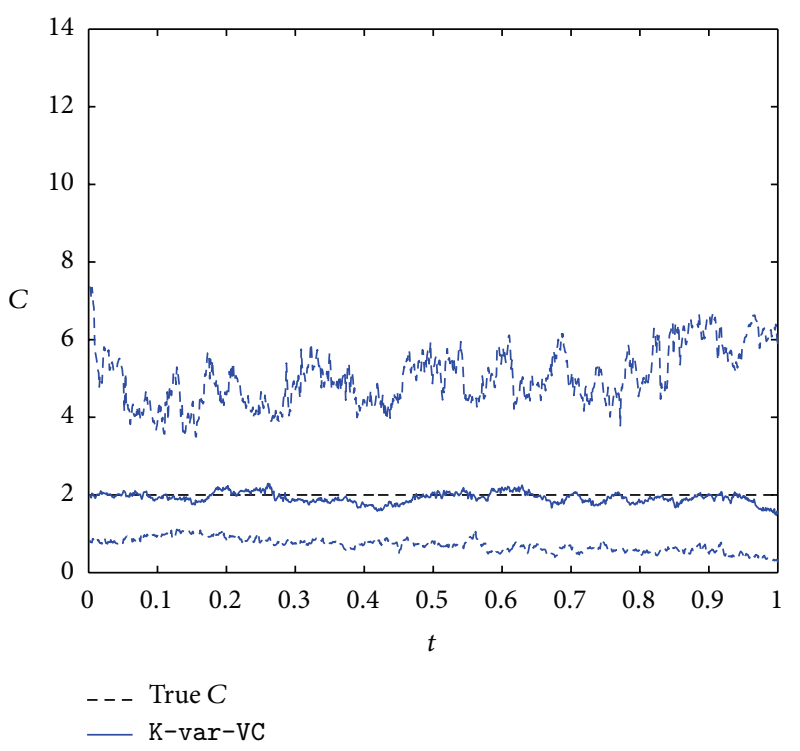

(a) $\mathrm{K}$-var-VC, no noise

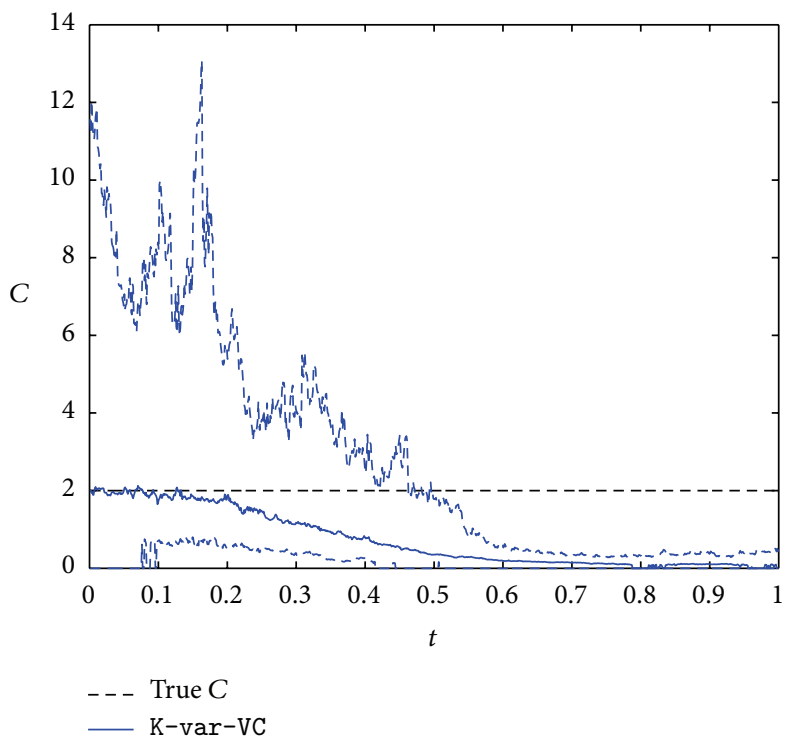

(c) K-var-VC, SNR 10

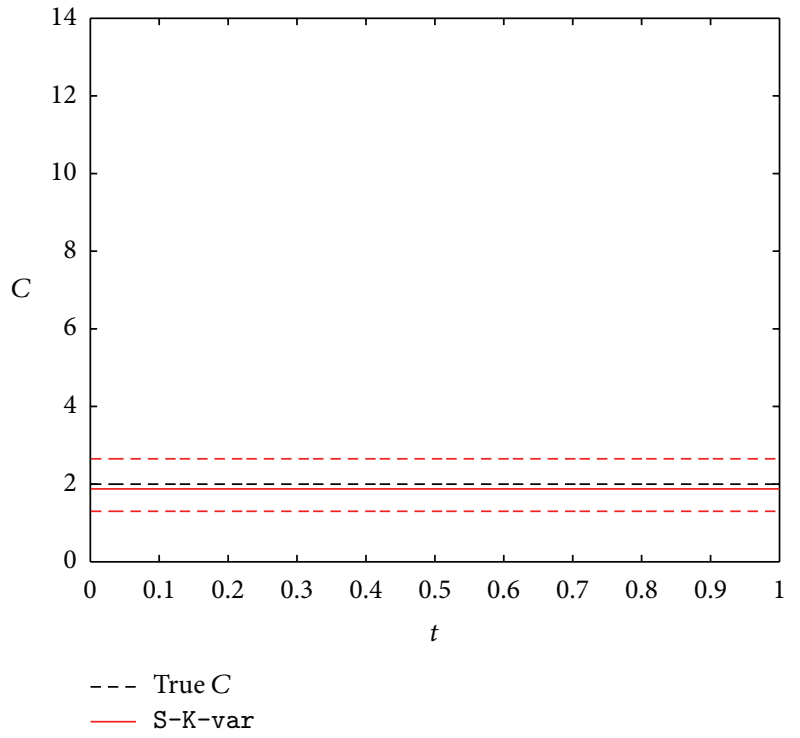

(b) S-K-var, no noise

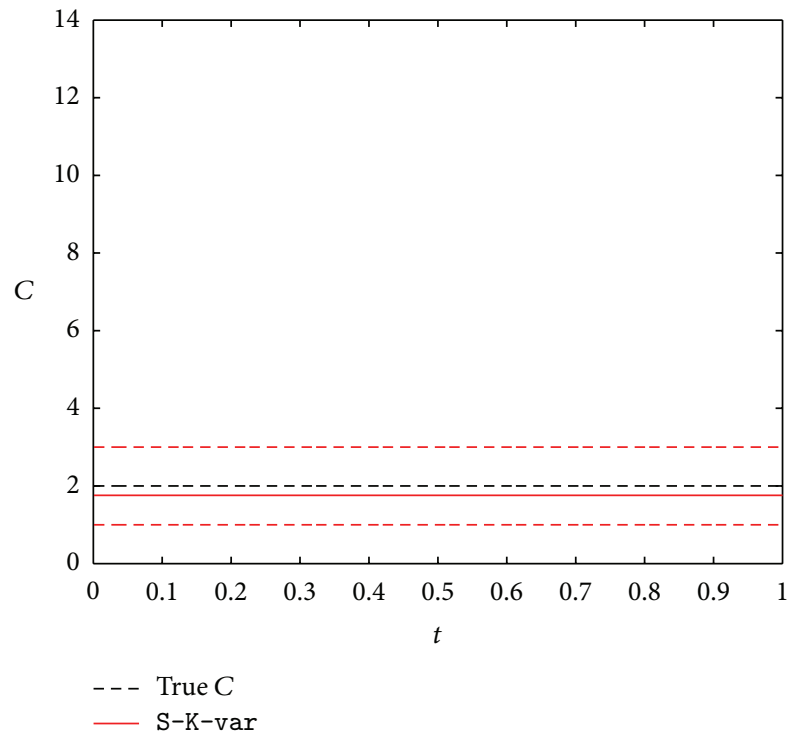

(d) S-K-var, SNR 10

FIGURE 3: Estimation of $C$ by $\mathrm{K}-\mathrm{var}-\mathrm{VC}$ with empirical $95 \%$ confidence intervals in blue is shown in (a) for no noise and (c) for SNR 10 when true $C=2$. Similarly, estimation of $C$ by S-K-var in red is shown in (b) for no noise and (d) for SNR 10. S-K-var yields more stable and shorter confidence intervals.

Db.i denoting a Daubechies wavelet filter of order $i$, and Sym. $i$ denoting a Symlet wavelet filter of order $i$. We generate 1,000 series of length $N=4096$ for step-function $H(t)$ and $N=1024$ for straight-line function $H(t)$. A simple difference filter [ $\left.\begin{array}{lll}1 & -2 & 1\end{array}\right]$ (Diff . 2) was used for straight-line $H(t)$, and $\mathrm{Db} .6$ was used for step-function $H(t)$. For the local spectra slope of LSS, the length of the subsignal was set to be 512 , which is sufficient for its numerical stability, and the two levels, by which spectral slopes are calculated, were 3 and 7. The size of a neighborhood of $t, v(t)$ in (8), is set to be 50 for $\mathrm{S}-\mathrm{K}$-var, $\mathrm{K}$-var, and $\mathrm{K}$-var-VC.

Illustrations of the estimators under no noise are shown in Figure 2. Estimation by $\mathrm{K}-\mathrm{var}-\mathrm{VC}$ most accurately follows true $H(t)$ among the tested methods. Estimation results by
$\mathrm{K}$-var, considering no scale parameter $C$, notably deviate from true $H(t)$. We note that the distance between $\mathrm{K}$-var estimation and true $H(t)$ relates to $C$. Estimators by LSS are bumpy because it assumes that subsignals during its computation follow $\mathrm{fBm}$ without considering the variability of $H(t)$. We also observe that $\mathrm{K}-\mathrm{var}-\mathrm{VC}$ is more unstable than $\mathrm{S}-\mathrm{K}$-var.

Regarding the estimation of $C$, the comparison between $\mathrm{K}-\mathrm{var}-\mathrm{VC}$ and $\mathrm{S}-\mathrm{K}-\mathrm{var}$ is shown in Figure 3, in which empirical confidence intervals for true $C=2$ are shown with the upper panels for no noise and the lower panels for SNR 10. We sampled 1000 series of $W^{\prime}(t)$ with $C=$ 2 and straight-line $H(t)$ under white noise of SNR 10. Consistently, the estimation results by $\mathrm{S}-\mathrm{K}-\mathrm{var}$ at the right 


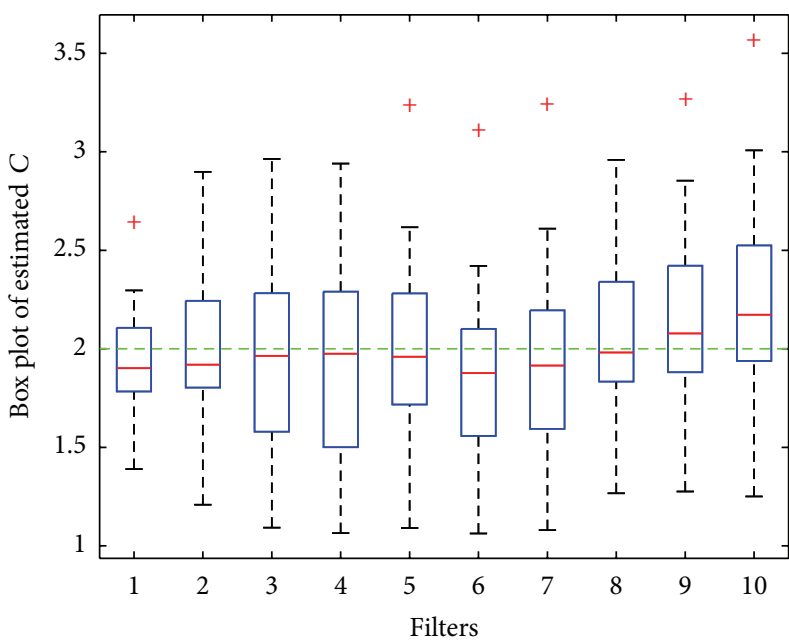

(a)

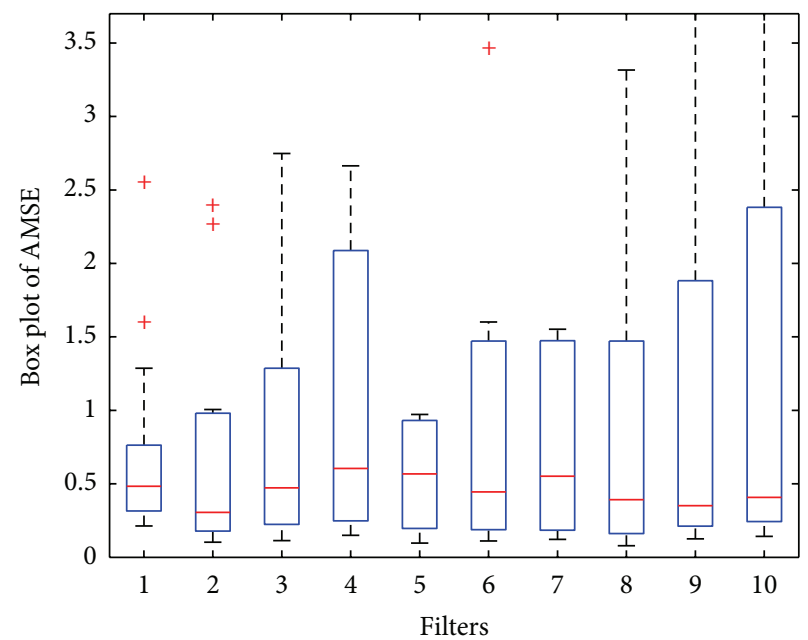

(b)

Figure 4: Performance according to different filters with straight-line $H(t)$; in panel (a), box plots for estimations of $C=2$; in (b), box plots for AMSE of $H(t)$. Filter numbers represent the following: 1, Diff . 1; 2, Diff . 2; 3, Diff . 3; 4, Diff . 4; 5, Sym. 4; 6, Sym. 6; 7, Sym. 8; 8, Db. 2; 9, $\mathrm{Db} .3$; and as well as $10, \mathrm{Db} .4$.

TABLE 1: Average mean squared error for the $\mathrm{S}-\mathrm{K}-\mathrm{var}, \mathrm{K}-\mathrm{var}-\mathrm{VC}$, and $\mathrm{K}$-var methods in various settings of variance constant $C$ and SNR levels based on 1000 sample paths of $W^{\prime}(t)$ for each of step-function and straight-line $H(t)$. The asterisk marks show that $\mathrm{S}-\mathrm{K}-\mathrm{var}$ outperformed the other methods.

\begin{tabular}{|c|c|c|c|c|c|c|}
\hline \multirow{3}{*}{ Parameters } & \multicolumn{6}{|c|}{ Average mean squared error } \\
\hline & \multicolumn{3}{|c|}{ Step-function $H(t)$} & \multicolumn{3}{|c|}{ Straight-line $H(t)$} \\
\hline & $\mathrm{S}-\mathrm{K}-\mathrm{var}$ & $\mathrm{K}$-var-VC & $\mathrm{K}$-var & $\mathrm{S}-\mathrm{K}-\mathrm{var}$ & $\mathrm{K}$-var-VC & $\mathrm{K}$-var \\
\hline$C=1$, SNR 10 & $31.54^{*}$ & 178.6 & 107.1 & 84.62 & 215.5 & 81.31 \\
\hline 30 & $22.85^{*}$ & 89.64 & 64.59 & 25.48 & 147.8 & 23.53 \\
\hline$\infty$ & $9.940^{*}$ & 26.31 & 48.16 & $.8242^{*}$ & 9.508 & .9376 \\
\hline$C=4$, SNR 10 & $15.56^{*}$ & 212.5 & 82.29 & $46.16^{*}$ & 176.9 & 175.2 \\
\hline 30 & $4.406^{*}$ & 152.2 & 39.91 & $36.11^{*}$ & 92.07 & 120.2 \\
\hline$\infty$ & $.9706^{*}$ & 10.00 & 8.173 & $10.04^{*}$ & 24.73 & 93.21 \\
\hline$C=10$, SNR 10 & $61.80^{*}$ & 178.2 & 281.5 & $89.94^{*}$ & 216.5 & 223.9 \\
\hline 30 & $55.97^{*}$ & 87.35 & 237.0 & $42.21^{*}$ & 150.9 & 144.6 \\
\hline$\infty$ & $33.86^{*}$ & 26.08 & 193.1 & $1.425^{*}$ & 9.288 & 77.32 \\
\hline
\end{tabular}

panels are more accurate, and their confidence intervals are sharper than those by $\mathrm{K}-\mathrm{var}-\mathrm{VC}$. We also note that the confidence intervals by $S-K-\operatorname{var}$ in Figures 3(b) and 3(d) are constant in time since $\mathrm{S}-\mathrm{K}$-var employs a global constant in regression model (16). A noise level of SNR 10 heavily worsened the estimation results by $\mathrm{K}-\mathrm{var}-\mathrm{VC}$ while those by $\mathrm{S}-\mathrm{K}$-var yielded a slight increase in the confidence intervals. Accurate estimation of variance level $C$ by $\mathrm{K}$-var-VC leads to accurate estimation of $H(t)$, which will be demonstrated in the following tests.

We compared $\mathrm{S}-\mathrm{K}$-var with $\mathrm{K}$-var-VC and $\mathrm{K}$-var in terms of AMSE in various settings. The method LSS was dropped due to obvious poor performance as is shown in Figure 2. We varied the levels of variance $C$ from 1 to 4 and 10 under SNR levels of 10, 30, and the infinity. The number of sample path $W^{\prime}(t)$ was 1000 in each setting, and AMSE was computed for each of the methods. The results are shown in Table 1 for each step-function and straightline $H(t)$. We observe that our proposed method S-K-var consistently outperforms the other methods except for only a few settings. Overall, there was little difference between $\mathrm{K}$-var-VC and $\mathrm{K}$-var in performance. This experimental result is not surprising since $\mathrm{S}-\mathrm{K}-\mathrm{var}$ reflects the existence of white noise and globally includes variance constant $C$.

The effects of adapted filters are summarized in Figure 4. The experiments were done with straight-line $H(t)$, variance $C=2$, and $\mathrm{SNR}=7$. We observe that the performance of $\mathrm{S}-\mathrm{K}$-var on the estimation of $C$ does not change much depending on the filter it uses. However, we mention that the variance of AMSEs tends to increase according to the filter size.

\section{Conclusion}

To conclude, we proposed the joint estimators of the timechanging Hurst exponent $H(t)$ and its variance coefficient $C$ for $\mathrm{mBm}$ under white noise. The proposed method is based on filtering sampled paths with dilated high-pass 
filters to derive regularity conditions at associated scales. The second empirical moment, average squared energy, of the filtered signals near a time position is connected to the theoretical expectation and used to establish a regression setup through the asymptotic distribution of multiscale local variation statistics. The effectiveness of the approach was verified through numerical experiments that compared it with that of several other approaches. Simulation results show that the proposed approach yields more precise and stable estimation of Hurst exponents and variance constants under noiseless or noised conditions.

\section{Appendices}

\section{A. Proof of Proposition 1}

Let $G(t)$ denote $C \sqrt{K(2 H(t)) / 2}$ for the sake of simplicity. Then, the covariance $\mathbb{E}\left[V_{\mathbf{a}}^{\prime}\left(j_{1} / N\right) V_{\mathbf{a}}^{\prime}\left(j_{2} / N\right)\right]$ becomes, by $u=$ $\lambda / N$,

$$
\begin{aligned}
& \sum_{p, q} a_{p} a_{q}\left(G\left(\frac{j_{1}-p}{N}\right) G\left(\frac{j_{2}-q}{N}\right)\right. \\
& \times \int \frac{\exp \left(\mathbf{i}\left(\left(j_{1}-p\right) / N\right) \lambda\right)-1}{|\lambda|^{H\left(\left(j_{1}-p\right) / N\right)+1 / 2}} \\
& \times \frac{\exp \left(-\mathbf{i}\left(\left(j_{2}-q\right) / N\right) \lambda\right)-1}{|\lambda|^{H\left(\left(j_{2}-q^{\prime}\right) / N\right)+1 / 2}} d \lambda \\
& \left.+\sigma^{2} \mathbb{E}\left[\varepsilon\left(\frac{j_{1}-p}{N}\right) \varepsilon\left(\frac{j_{2}-q}{N}\right)\right]\right) \\
& =\sum_{p, q} a_{p} a_{q} \frac{G\left(\left(j_{1}-p\right) / N\right) G\left(\left(j_{2}-q\right) / N\right)}{N^{H\left(\left(j_{1}-p\right) / N\right)+H\left(\left(j_{2}-q\right) / N\right)}} \\
& \times \int \frac{\exp \left(\mathbf{i}\left(j_{1}-p\right) u\right)-1}{|u|^{H\left(\left(j_{1}-p\right) / N\right)+1 / 2}} \times \frac{\exp \left(-\mathbf{i}\left(j_{2}-q\right) u\right)-1}{|u|^{H\left(\left(j_{2}-q\right) / N\right)+1 / 2}} d u \\
& +\sigma^{2} \sum_{p-q=j_{1}-j_{2}} a_{p} a_{q} .
\end{aligned}
$$

By Taylor's expansion and Hölderian order $\eta$ of $H(t)$, for $j_{1}-p$ in the neighborhood of $t_{1}$ we approximate $G\left(\left(j_{1}-p\right) / N\right)$ with

$$
\begin{aligned}
G\left(\frac{j_{1}-p}{N}\right) & =G\left(t_{1}\right)+\mathcal{O}\left(\left|H\left(\frac{j_{1}-p}{N}\right)-H\left(t_{1}\right)\right|\right) \\
& =G\left(t_{1}\right)+\mathcal{O}\left(\epsilon^{\eta}\right) .
\end{aligned}
$$

Similarly, $G\left(\left(j_{2}-q\right) / N\right)$ is approximated with $G\left(\left(j_{2}-\right.\right.$ $q) / N)=G\left(t_{2}\right)+\mathcal{O}\left(\epsilon^{\eta}\right)$. In addition, by Taylor's expansion of $N^{x} / \log (N) N^{k}$ around the point $k, N^{x} / \log (N) N^{k}=$ $1 / \log (N)+\mathcal{O}(|x-k|)$, we have

$$
\begin{aligned}
& \frac{1}{N^{H\left(\left(j_{1}-p\right) / N\right)+H\left(\left(j_{2}-q\right) / N\right)}}=\frac{1}{N^{H\left(t_{1}\right)+H\left(t_{2}\right)}}\left(1+\mathcal{O}\left(\epsilon^{\eta} \log N\right)\right), \\
& \frac{1}{|u|^{H\left(\left(j_{1}-p\right) / N\right)+H\left(\left(j_{2}-q\right) / N\right)}}=\frac{1}{|u|^{H\left(t_{1}\right)+H\left(t_{2}\right)}}\left(1+\mathcal{O}\left(\epsilon^{\eta} \log u\right)\right) .
\end{aligned}
$$

Using also $\epsilon^{\eta} \rightarrow 0$ as $N$ goes to infinity, the covariance can be written as follows:

$$
\begin{aligned}
& \sum_{p, q} a_{p} a_{q} \frac{G\left(t_{1}\right) G\left(t_{2}\right)}{N^{H\left(t_{1}\right)+H\left(t_{2}\right)}} \\
& \times \int \frac{\exp \left(\mathbf{i}\left(j_{1}-p\right) u\right)-1}{|u|^{H\left(t_{1}\right)+1 / 2}} \times \frac{\exp \left(-\mathbf{i}\left(j_{2}-q\right) u\right)-1}{|u|^{H\left(t_{2}\right)+1 / 2}} d u \\
& +\sigma^{2} \sum_{p-q=j_{1}-j_{2}} a_{p} a_{q}+\mathcal{O}\left(\epsilon^{\eta} \log N\right) .
\end{aligned}
$$

Since the order of filter $\mathbf{a}$ is at least $1, \sum_{q} a_{q}=0$, (A.4) becomes

$$
\begin{aligned}
& \sum_{p, q} a_{p} a_{q} \frac{G\left(t_{1}\right) G\left(t_{2}\right)}{N^{H\left(t_{1}\right)+H\left(t_{2}\right)}} \int \frac{\cos \left(\left(j_{1}-p-j_{2}+q\right) u\right)-1}{|u|^{H\left(t_{1}\right)+H\left(t_{2}\right)+1}} d u \\
& +\sigma^{2} \sum_{p-q=j_{1}-j_{2}} a_{p} a_{q}+\mathcal{O}\left(\epsilon^{\eta} \log N\right) .
\end{aligned}
$$

Since $K(\alpha) \int\left((1-\cos (\kappa u)) /|u|^{\alpha+1}\right) d u=|\kappa|^{\alpha}$, for all $\kappa, 0<\alpha<$ 2 ,

$$
\begin{aligned}
\mathbb{E}\left[V_{\mathbf{a}}^{\prime}\left(\frac{j_{1}}{N}\right) V_{\mathbf{a}}^{\prime}\left(\frac{j_{2}}{N}\right)\right]= & -\sum_{p, q} a_{p} a_{q} \frac{G\left(t_{1}\right) G\left(t_{2}\right)}{N^{H\left(t_{1}\right)+H\left(t_{2}\right)}} \\
& \times \frac{\left|j_{1}-p-j_{2}+q\right|^{H\left(t_{1}\right)+H\left(t_{2}\right)}}{K\left(H\left(t_{1}\right)+H\left(t_{2}\right)\right)} \\
& +\sigma^{2} \sum_{p-q=j_{1}-j_{2}} a_{p} a_{q}+\mathcal{O}\left(\epsilon^{\eta} \log N\right) \\
= & -\frac{C^{2} g\left(H\left(t_{1}\right), H\left(t_{2}\right)\right)}{N^{H\left(t_{1}\right)+H\left(t_{2}\right)}} \\
& \times \sum_{p, q} \frac{a_{p} a_{q}}{2}\left|j_{1}-p-j_{2}+q\right|^{H\left(t_{1}\right)+H\left(t_{2}\right)} \\
& +\sigma^{2} \sum_{p-q=j-j^{\prime}} a_{q} a_{q}^{\prime}+\mathcal{O}\left(\epsilon^{\eta} \log N\right) \\
= & \frac{C^{2} g\left(H\left(t_{1}\right), H\left(t_{2}\right)\right)}{N^{H\left(t_{1}\right)+H\left(t_{2}\right)}} \\
& \times \pi_{\mathrm{a}, H\left(t_{1}\right) / 2+H\left(t_{2}\right) / 2}\left(j_{1}-j_{2}\right) \\
& +\sigma^{2} \sum_{p-q=j_{1}-j_{2}} a_{p} a_{q}+\mathcal{O}\left(\epsilon^{\eta} \log N\right),
\end{aligned}
$$


where $\pi_{\mathbf{a}, h}(k)=-(1 / 2) \sum_{p, q=0}^{l} a_{p} a_{q}|k-p+q|^{2 h}$. When we replace $\mathbf{a}$ with $\mathbf{a}^{(m)}$, the proof is completed.

\section{B. Proof of Proposition 2}

Let $A$ and $B$ denote, respectively,

$$
\begin{gathered}
A=\frac{1}{|\nu(t)|} \sum_{j \in v(t)} \sum_{p, q}^{l} a_{p} a_{q} W\left(\frac{j-p}{N}\right) \varepsilon\left(\frac{j-q}{N}\right), \\
B=\frac{1}{|\nu(t)|} \sum_{j \in v(t)}\left(\sum_{q}^{l} a_{q} \varepsilon\left(\frac{j-q}{N}\right)\right)^{2} .
\end{gathered}
$$

Then $\log S^{\prime}(t, \mathbf{a})$ can be written as

$$
\begin{aligned}
\log S^{\prime}(t, \mathbf{a})= & \log \frac{1}{|\nu(t)|} \sum_{j \in \nu(t)} V_{\mathbf{a}}^{\prime}\left(\frac{j}{N}\right)^{2} \\
= & \log \frac{1}{|\nu(t)|} \\
& \times \sum_{j \in v(t)}\left(\left(\sum_{q}^{l} a_{q} W\left(\frac{j-q}{N}\right)\right)^{2}\right. \\
& +2 \sigma \sum_{p, q}^{l} a_{p} a_{q} W\left(\frac{j-p}{N}\right) \varepsilon\left(\frac{j-q}{N}\right) \\
& \left.+\sigma^{2}\left(\sum_{q}^{l} a_{q} \varepsilon\left(\frac{j-q}{N}\right)\right)^{2}\right) \\
= & \log S(t, \mathbf{a})+\left(2 \sigma \frac{A}{S(t, \mathbf{a})}+\sigma^{2} \frac{B}{S(t, \mathbf{a})}\right) \\
& \times(1+o(1))
\end{aligned}
$$

by Taylor's expansion of $\log (1+x)=x(1+o(1))$ near $x=0$. Similarly, $\log \mathbb{E}\left[S^{\prime}(t, \mathbf{a})\right]$ is expressed as

$$
\begin{aligned}
\log \mathbb{E}\left[S^{\prime}(t, \mathbf{a})\right]= & \log \left(\mathbb{E}[S(t, \mathbf{a})]+\sigma^{2} \sum_{q}^{l} a_{q}^{2}\right) \\
= & \log (\mathbb{E}[S(t, \mathbf{a})])+\left(\frac{\sigma^{2} \sum_{q}^{l} a_{q}^{2}}{\mathbb{E}[S(t, \mathbf{a})]}\right) \\
& \times(1+o(1)) .
\end{aligned}
$$

Using independence of $W(t)$ and $\varepsilon(t)$, the property of white noise $\varepsilon(t)$, and the convergence of $S(t, \mathbf{a})$ to $\mathbb{E}[S(t, \mathbf{a})]$ almost surely as $N \rightarrow \infty$, we approximate $A \rightarrow 0, B \rightarrow$ $\sum_{q}^{l} a_{q}^{2}$, and $\sigma^{2}(B / S(t, \mathbf{a})) \rightarrow \sigma^{2}\left(\sum_{q}^{l} a_{q}^{2} / \mathbb{E}[S(t, \mathbf{a})]\right)$. Then the difference between $\log S^{\prime}(t, \mathbf{a})$ in (B.2) and $\log \mathbb{E}\left[S^{\prime}(t, \mathbf{a})\right]$ in (B.3) becomes

$$
\begin{aligned}
& \log S^{\prime}(t, \mathbf{a})-\log \mathbb{E}\left[S^{\prime}(t, \mathbf{a})\right] \\
& =\log S(t, \mathbf{a})-\log \mathbb{E}[S(t, \mathbf{a})]+o(1) \\
& =\log \left(\frac{1}{|\nu(t)|} \sum_{j \in v(t)} \frac{V_{\mathbf{a}}(j / N)^{2}}{\mathbb{E}[S(t, \mathbf{a})]}\right)+o(1) \\
& \quad(\text { by the definition of } S(t, \mathbf{a})) \\
& =\log \left(\frac{1}{|\nu(t)|} \sum_{j \in v(t)} \frac{V_{\mathbf{a}}(j / N)^{2}}{\mathbb{E}\left[V_{\mathbf{a}}(j / N)^{2}\right]}\right)+o(1) \\
& =\left(\left(\frac{1}{|\nu(t)|} \sum_{j \in v(t)} \frac{V_{\mathbf{a}}(j / N)^{2}}{\mathbb{E}\left[V_{\mathbf{a}}(j / N)^{2}\right]}\right)-1\right)(1+o(1)) \\
& =V(t, \mathbf{a})(1+o(1)) . \quad\left(\text { by the stationarity of } V_{\mathbf{a}}\right)
\end{aligned}
$$

\section{Conflict of Interests}

The authors declare that there is no conflict of interests regarding the publication of this paper.

\section{Acknowledgments}

This research was provided by Hanyang University (201300000001465). The authors give special thanks to Brani Vidakovic and Jean-Francois Coeurjolly for their careful comments.

\section{References}

[1] B. B. Mandelbrot and J. W. Van Ness, "Fractional Brownian motions, fractional noises and applications," SIAM Review, vol. 10, pp. 422-437, 1968.

[2] M. Li, Y. Chen, J. Y. Li, and W. Zhao, "Hölder scales of sea level," Mathematical Problems in Engineering, vol. 2012, Article ID 863707, 22 pages, 2012.

[3] S. V. Muniandy, S. C. Lim, and R. Murugan, "Inhomogeneous scaling behaviors in Malaysian foreign currency exchange rates," Physica A, vol. 301, no. 1-4, pp. 407-428, 2001.

[4] R. H. Riedi, M. S. Crouse, V. J. Ribeiro, and R. G. Baraniuk, "A multifractal wavelet model with application to network traffic," IEEE Transactions on Information Theory, vol. 45, no. 3, pp. 9921018, 1999.

[5] M. Li, W. Zhao, and S. Chen, "mBm-based scalings of traffic propagated in internet," Mathematical Problems in Engineering, vol. 2011, Article ID 389803, 21 pages, 2011.

[6] M. Li and W. Zhao, "Quantitatively investigating locally weak stationarity of modified multifractional gaussian noise," Physica A, vol. 391, no. 24, pp. 6268-6278, 2012. 
[7] J. Lévy-Véhel and R. Peltier, "Multifractional brownian motion: definition and preliminary results," Rapport de Recherche de L'INRIA n2645, 1995.

[8] A. Benassi, S. Cohen, and J. Istas, "Identifying the multifractional function of a gaussian process," Statistics \& Probability Letters, vol. 39, no. 4, pp. 337-345, 1998.

[9] S. C. Lim and S. V. Muniandy, "On some possible generalizations of fractional Brownian motion," Physics Letters. A, vol. 266, no. 2-3, pp. 140-145, 2000.

[10] S. V. Muniandy and S. C. Lim, "Modeling of locally self-similar processes using multifractional brownian motion of RiemannLiouville type," Physical Review E, vol. 63, no. 4, pp. 461041461047, 2001.

[11] J. Istas and G. Lang, "Quadratic variations and estimation of the local Hölder index of a Gaussian process," Annales de l'Institut Henri Poincaré B, vol. 33, no. 4, pp. 407-436, 1997.

[12] J. T. Kent and A. T. A. Wood, "Estimating the fractal dimension of a locally self-similar gaussian process by using increments," Journal of the Royal Statistical Society B, vol. 59, no. 3, pp. 679699, 1997.

[13] J. F. Coeurjolly, "Estimating the parameters of a fractional brownian motion by discrete variations of its sample paths," Statistical Inference for Stochastic Processes, vol. 4, no. 2, pp. 199227, 2001.

[14] M. Fhima, A. Guillin, and P. R. Bertrand, "Fast change point analysis on the Hurst index of piecewise fractional Brownian motion," http://arxiv.org/abs/1103.4029.

[15] J. Beran, Statistics for Long-Memory Processes, vol. 61 of Monographs on Statistics and Applied Probability, Chapman \& Hall, London, UK, 1994.

[16] J.M. Bardet and P. Bertrand, "Definition, properties and wavelet analysis of multiscale fractional Brownian motion," Fractals, vol. 15, no. 1, pp. 73-87, 2007.

[17] J.F. Coeurjolly, "Identification of multifractional brownian motion," Bernoulli, vol. 11, no. 6, pp. 987-1008, 2005.

[18] A. Shapiro, "Statistical inference of moment structures," in Handbook of Latent Variable and Related Models, pp. 229-260, 2007.

[19] I. Daubechies, Ten Lectures on Wavelets, vol. 61 of CBMSNSF Regional Conference Series in Applied Mathematics, SIAM, Philadelphia, Pa, USA, 1992.

[20] B. Vidakovic, Statistical Modeling by Wavelets, Wiley Series in Probability and Statistics: Applied Probability and Statistics, John Wiley \& Sons, Hoboken, NJ, USA, 1999.

[21] J. Gao, Multiscale Analysis of Complex Time Series: Integration of Chaos and Random Fractal Theory, and Beyond, John Wiley \& Sons, Hoboken, NJ, USA, 2007.

[22] A. Wood and G. Chan, "Simulation of stationary gaussian processes in $[0,1]$ d," Journal of Computational and Graphical Statistics, vol. 3, no. 4, pp. 409-432, 1994. 


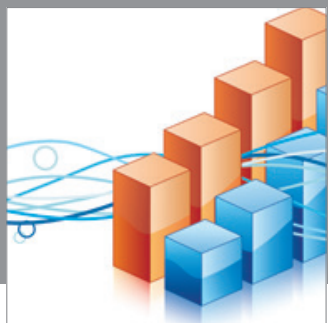

Advances in

Operations Research

mansans

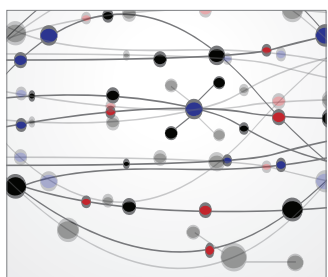

The Scientific World Journal
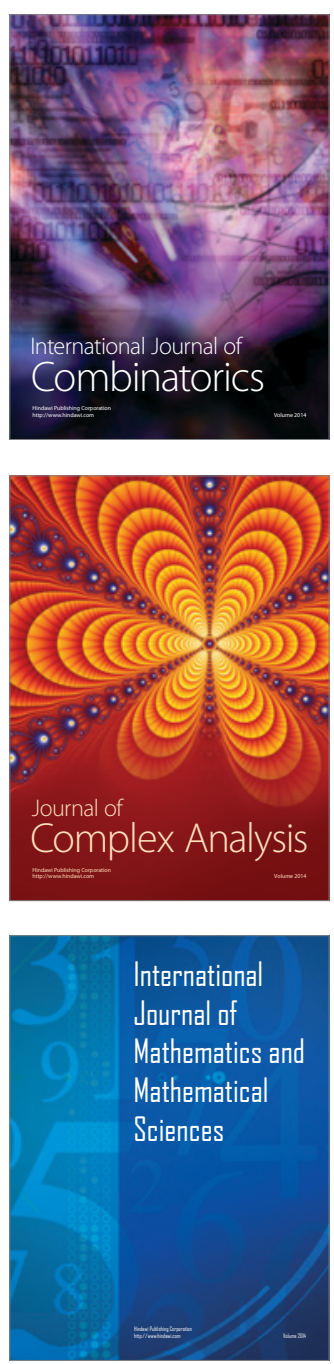
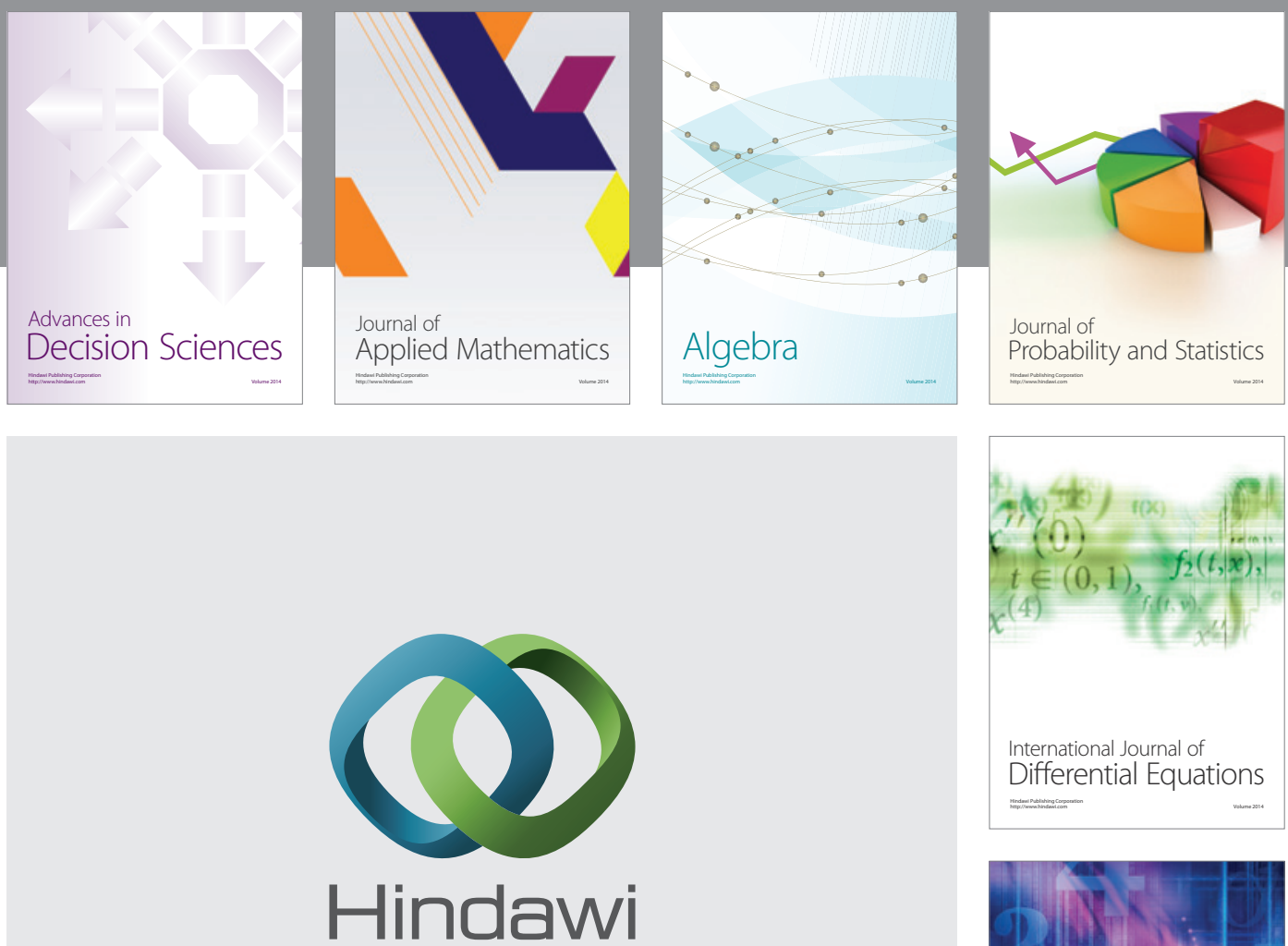

Submit your manuscripts at http://www.hindawi.com
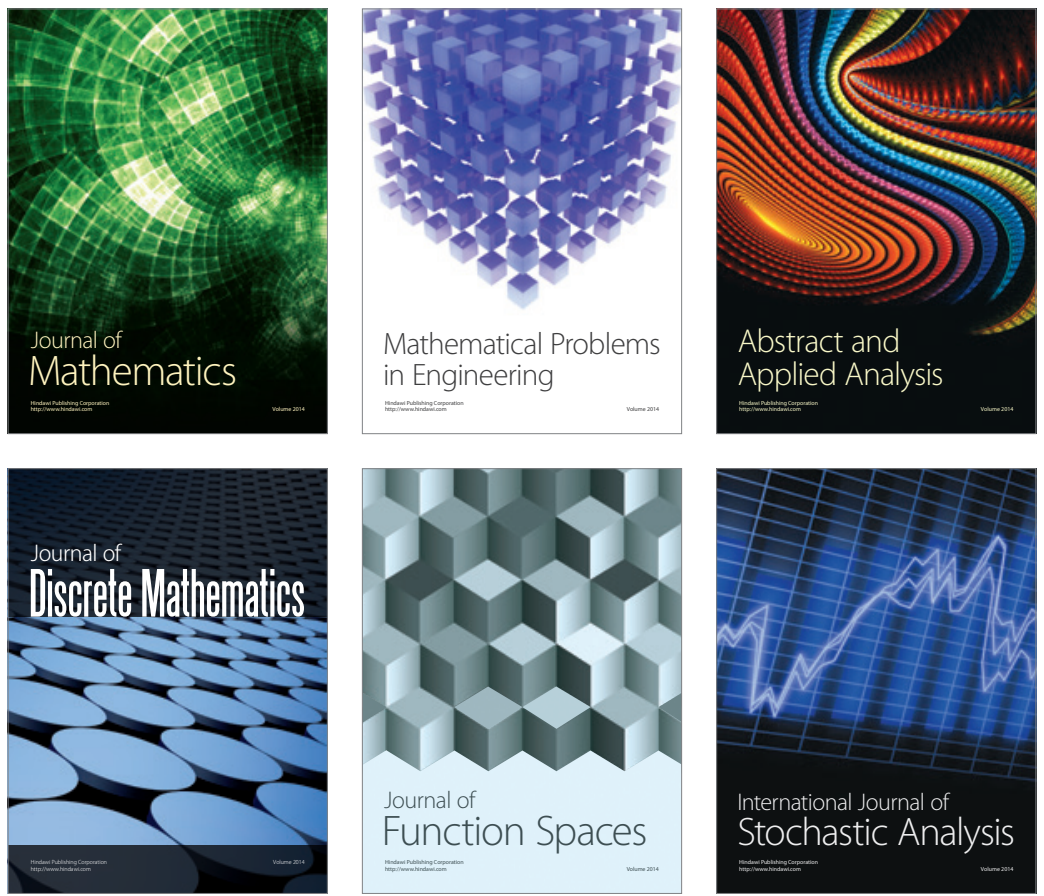

Journal of

Function Spaces

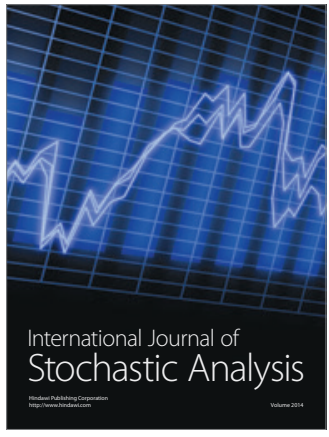

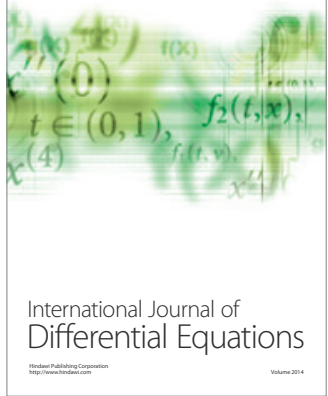
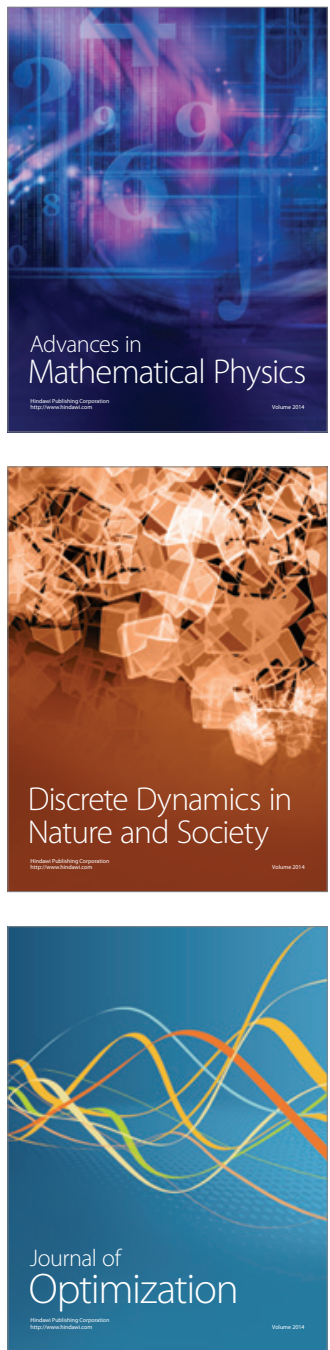